PROCEEDINGS OF THE

AMERICAN MATHEMATICAL SOCIETY

Volume 129, Number 7, Pages 2135-2140

S 0002-9939(00)05998-0

Article electronically published on December 13, 2000

\title{
NECESSARY AND SUFFICIENT CONDITIONS FOR S-HOPFIAN MANIFOLDS TO BE CODIMENSION-2 FIBRATORS
}

\author{
YOUNG HO IM AND YONGKUK KIM
}

(Communicated by Ralph Cohen)

\begin{abstract}
Fibrators help detect approximate fibrations. A closed, connected $n$-manifold $N$ is called a codimension-2 fibrator if each map $p: M \rightarrow B$ defined on an $(n+2)$-manifold $M$ such that all fibre $p^{-1}(b), b \in B$, are shape equivalent to $N$ is an approximate fibration. The most natural objects $N$ to study are s-Hopfian manifolds. In this note we give some necessary and sufficient conditions for s-Hopfian manifolds to be codimension-2 fibrators.
\end{abstract}

Approximate fibrations, introduced and extensively studied by Coram and Duvall, form an important class of mappings, almost as efficacious as Hurewicz fibrations and cell-like maps. The advantage of this notion is that on one hand there exists an exact homotopy sequence but on the other hand there are more such approximate fibrations available. (See 3,4 for the definition and usefulness of approximate fibrations.) Fibrators help detect approximate fibrations. A closed, connected $n$-manifold $N$ is called a codimension-2 fibrator if each map $p: M \rightarrow B$ defined on an $(n+2)$-manifold $M$ such that all fibres $p^{-1}(b), b \in B$, are shape equivalent to $N$ is an approximate fibration. The most natural objects $N$ to study are s-Hopfian manifolds. All closed s-Hopfian manifolds with either trivial fundamental group or Hopfian fundamental group and nonzero Euler characteristic or hyperhopfian fundamental group are known to be codimension-2 fibrators [6, 7, 15, 16, 18]. Surprisingly few nonfibrators are known so far. The best well-known nonfibrators are closed manifolds that cyclically cover themselves nontrivially, such as $S^{1} \times S^{1}$ and $\mathbb{R} P^{n} \# \mathbb{R} P^{n}[\underline{6}$, Theorem 4.2$]$. Recently Daverman gave some other kind of nonfibrator, which is $L(p, q) \times S^{3}$ [8] Example 2.1]. None of them gives necessary and sufficient conditions for s-Hopfian manifolds to be codimension-2 fibrators in general cases. In this note we give some necessary and sufficient conditions in some special cases.

Received by the editors October 19, 1999.

2000 Mathematics Subject Classification. Primary 57N15, 55M25; Secondary 57M10, 54B15.

Key words and phrases. Codimension-2 fibrator, s-Hopfian manifold, Hopfian group, approximate fibration.

The first author's research was supported by Korea Research Foundation Grant (KRF-2000041-D00023).

The second author's research was supported by Korea Research Foundation Grant (KRF-2000015-DP0034).

(C)2000 American Mathematical Society 


\section{DEFINITIONS AND PRELIMINARIES}

Throughout this paper, the symbols $\simeq$ and $\cong$ denote a homotopy equivalence and an isomorphism, respectively, and the symbols $\chi$ and $\beta_{i}$ are used to denote Euler characteristic and the $i$-th betti number, respectively. All manifolds are understood to be finite dimensional, connected and metric.

Let $N$ be a closed manifold. A proper map $p: M \longrightarrow B$ is $N$ - like if each fiber $p^{-1}(b)$ is shape equivalent to $N$. For simplicity, we shall assume that each fiber $p^{-1}(b)$ in an $N$-like map to be an $A N R$ having the homotopy type of $N$.

Suppose that $N$ is a closed $n$-manifold and a proper map $p: M \longrightarrow B$ is $N$-like. Let $G$ be the set of all fibers, i.e., $G=\left\{p^{-1}(b): b \in B\right\}$. Put $C=\{p(g) \in B: g \in G$ and there exist a neighborhood $U_{g}$ of $g$ in $M$ and a retraction $R_{g}: U_{g} \longrightarrow g$ such that $R_{g} \mid g^{\prime}: g^{\prime} \longrightarrow g$ is a degree one map for all $g^{\prime} \in G$ with $g^{\prime} \in G$ in $\left.U_{g}\right\}$, and $C^{\prime}=\left\{p(g) \in B: g \in G\right.$ and there exist a neighborhood $U_{g}$ of $g$ in $M$ and a retraction $R_{g}: U_{g} \longrightarrow g$ such that $R_{g} \mid g^{\prime}: g^{\prime} \longrightarrow g$ is a degree one mod 2 map for all $g^{\prime} \in G$ with $g^{\prime} \in G$ in $U_{g}$ \}. Call $C$ the continuity set of $p$ and $C^{\prime}$ the mod 2 continuity set of $p$. D. Coram and P. Duvall showed that $C$ and $C^{\prime}$ are dense, open subsets of $B[5]$.

Call a closed manifold $N$ Hopfian if it is orientable and every degree one map $N \longrightarrow N$ which induces a $\pi_{1}$-isomorphism is a homotopy equivalence. A closed manifold $N$ is s-Hopfian if $N$ is Hopfian when $N$ is orientable and $N_{H}$ is Hopfian when $N$ is non-orientable, where $N_{H}$ is the covering space of $N$ corresponding to $H=\bigcap_{i \in I}\left\{H_{i}:\left[\pi_{1}(N): H_{i}\right]=2\right\}$. All closed manifolds with nilpotent or finite fundamental group, all closed aspherical manifolds, and all closed $n$-manifolds $(n \leq 4)$ are examples of s-Hopfian manifolds. Whether all closed manifolds are sHopfian is related to the famous old problem of Hopf.

A group $\Gamma$ is said to be Hopfian if every epimorphism $f: \Gamma \rightarrow \Gamma$ is necessarily an isomorphism. The term aids in efficiently identifying approximate fibrations. A finitely presented group $\Gamma$ is said to be hyperhopfian if every homomorphism $f: \Gamma \longrightarrow \Gamma$ with $f(\Gamma)$ normal and $\Gamma / f(\Gamma)$ cyclic is an isomorphism (onto). Every Hopfian perfect group, every nonabelian group of order $p q$ with distinct primes $p$ and $q$, and the fundamental group of any closed surface with negative Euler characteristic are examples of hyperhopfian groups [7.

The next proposition plays an important role in this paper.

Proposition 1.1 ([7] Corollary 4.12]). If $\Gamma_{1}, \Gamma_{2}$ are nontrivial, finitely generated groups such that $\Gamma_{2} \neq \mathbb{Z}_{2}$ and $\Gamma_{1} * \Gamma_{2}$ is Hopfian, then $\Gamma_{1} * \Gamma_{2}$ is hyperhopfian.

The following is basic for investigating codimension-2 fibrators.

Proposition 1.2 ([6] and [17, Proposition 2.3]). Let $N$ be an s-Hopfian $n$-manifold with Hopfian fundamental group. Let a proper map $p: M \rightarrow B$ defined on an $(n+2)-$ manifold $M$ be $N$-like. Then, $p$ is an approximate fibration over some dense open subset $O$ of the mod 2 continuity set $C^{\prime}$ of $p$ and $C^{\prime} \backslash O$ is locally finite. Moreover, $C^{\prime}$ is dense open in int $B$ and int $B \backslash C^{\prime}$ is locally finite.

\section{NeCESSARY AND SUfFICIENT CONDITIONS FOR BEING CODIMENSION-2 FIBRATORS}

We use $N=N_{1}^{n} \# N_{2}^{n}$ to denote a connected sum of two $n$-manifolds $N_{1}$ and $N_{2}(n \geq 3)$, where neither $N_{1}$ nor $N_{2}$ is a homotopy sphere. Here, we note that $\pi_{1}(N) \cong \pi_{1}\left(N_{1}\right) * \pi_{1}\left(N_{2}\right)$ by the van Kampen Theorem. 
Lemma 2.1 ([17, Proposition 3.5]). Let $N=N_{1} \# N_{2}$ be an s-Hopfian closed $n$ manifold $(n \geq 3)$ with trivial $\pi_{1}\left(N_{1}\right)$ and Hopfian $\pi_{1}\left(N_{2}\right)$. If $\beta_{j}\left(N_{1}\right) \neq 0$ for some $j(0<j<n)$, then $N$ is a codimension-2 fibrator.

The next theorem is the orientation-free version of [17, Theorem 3.6].

Theorem 2.2. Let $N=N_{1} \# N_{2}$ be a closed 4-manifold with Hopfian $\pi_{1}\left(N_{i}\right)$ for $i=1,2$. Then $N$ is a codimension-2 fibrator, with only one exception $\mathbb{R} P^{4} \# \mathbb{R} P^{4}$ up to homotopy type.

Proof. First note that $N$ is an s-Hopfian manifold with Hopfian $\pi_{1}(N)$. We only need to consider the following two cases, otherwise $\pi_{1}(N)$ would be hyperhopfian or trivial.

Case (1): $\pi_{1}\left(N_{1}\right)$ is trivial and $\pi_{1}\left(N_{2}\right)$ is non-trivial.

Then $H_{2}\left(N_{1}\right)$ must be a free group so that $\beta_{2}\left(N_{1}\right) \neq 0$, otherwise $N_{1}$ is the homotopy sphere. Now apply to Lemma 2.1.

Case (2): $\pi_{1}\left(N_{i}\right)=\mathbb{Z}_{2}$ for each $i=1,2$.

Note that $\chi\left(U_{i}\right)=2+\beta_{2}\left(U_{i}\right) \geq 2$, where $U_{i}$ is the universal covering of $N_{i}$, so that $\chi\left(N_{i}\right) \geq 1$, for $\chi\left(U_{i}\right)=2 \chi\left(N_{i}\right)$. Since $N_{1} \# N_{2} \nsucceq \mathbb{R} P^{4} \# \mathbb{R} P^{4}, N_{1} \nsucceq \mathbb{R} P^{4}$ or $N_{2} \not \mathbb{R} P^{4}$. Say $N_{1} \nsucceq \mathbb{R} P^{4}$. Then $\chi\left(N_{1}\right) \neq 1$ (see [12, Lemma 1, p 130]). So $\chi(N)=\chi\left(N_{1}\right)-1+\chi\left(N_{2}\right)-1>0$.

Now we are going to prove that $\mathbb{R} P^{n} \# \mathbb{R} P^{n}$, up to homotopy type, is the only one exception for s-Hopfian $n$-manifolds $(n \geq 5)$ to be codimension-2 fibrators in the connected sum case.

Lemma 2.3. Let $N=N_{1} \# N_{2}$ be a closed s-Hopfian $n$-manifold $(n \geq 5)$ with $\pi_{1}\left(N_{i}\right) \cong \mathbb{Z}_{2}(i=1,2)$ and let $p: M \rightarrow \mathbb{R}^{2}$ be a proper, $N$-like map defined on an $(n+2)$-manifold that restricts to an approximate fibration over $\mathbb{R}^{2} \backslash \mathbf{0}$. If $N_{1}$ does not have the homotopy type of real projective $n$-space $\mathbb{R} P^{n}$, then $p$ is an approximate fibration over $\mathbb{R}^{2}$.

Proof. Use $g_{0}$ to represent $p^{-1}(\mathbf{0})$, which is a strong deformation retract of $M$ under a retraction $R: M \rightarrow g_{0}$. Pass to the covering $q: M_{H} \rightarrow M$ determined by $H=\bigcap_{i \in I}\left\{H_{i}:\left[\pi_{1}(N): H_{i}\right]=2\right\}$ and for any $x(\neq \mathbf{0}) \in \mathbb{R}^{2}$ consider the following diagram:

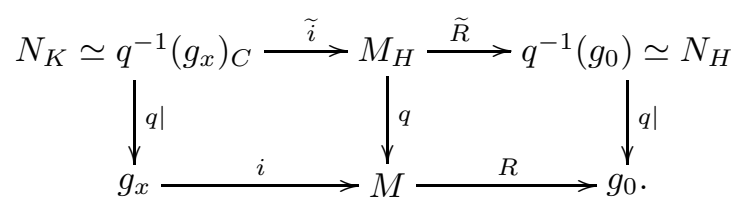

Here, $\widetilde{i}$ and $\widetilde{R}$ are liftings of $i \circ q \mid$ and $R \circ q$, respectively, and $q^{-1}\left(g_{x}\right)_{C}$ is the component of $q^{-1}\left(g_{x}\right)$. Note that $N_{K}$ is the covering of $N$ corresponding to $K=$ $i_{\#}^{-1}\left(q_{\#}\left(\pi_{1}\left(M_{H}\right)\right)\right)=i_{\#}^{-1}(H)$ (see [16]).

It suffices to show that $\widetilde{i}$ induces a $\pi_{1}$-epimorphism [10, Lemma 5.5]. Suppose not. Take the covering $\Theta: M^{*} \rightarrow M_{H}$ corresponding to $\widetilde{i}_{\#}\left(\pi_{1}\left(q^{-1}\left(g_{x}\right)_{C}\right)\right)$. Now 
consider

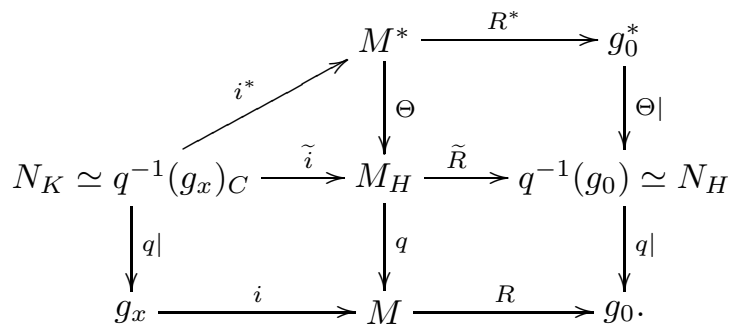

Here, $i^{*}$ and $R^{*}$ are liftings of $\widetilde{i}$ and $\widetilde{R} \circ \Theta$, respectively.

Note that $N_{H}$ is of the form

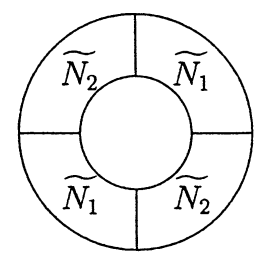

and $N_{K}$ is formed either by $\widetilde{N_{2}} \# N_{1} \# \widetilde{N_{2}}$ or by $\widetilde{N_{1}} \# N_{2} \# \widetilde{N_{1}}$ or by

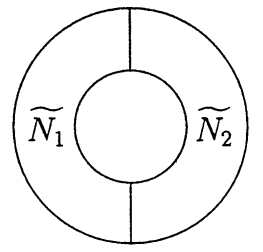

where $\widetilde{N}_{i}$ is the universal covering of $N_{i}$, respectively $(i=1,2)$.

Since $N_{1}$ does not have homotopy type of real projective $n$-space, the universal covering $\widetilde{N}_{1}$ of $N_{1}$ is not a homology sphere (see [2, 11, 19]) so that $H_{k}\left(\widetilde{N_{1}}\right) \neq 0$ for some $0<k<n$. However since the degree of $R^{*} \circ i^{*}$ is one [10, Lemma 5.5], for all $t,\left(R^{*} \circ i^{*}\right)_{*}: H_{t}\left(g_{x}\right) \rightarrow H_{t}\left(g_{0}^{*}\right)$ is an epimorphism, which is impossible, for $H_{1}\left(\widetilde{N_{2}} \# N_{1} \# \widetilde{N_{2}}\right) \cong H_{1}\left(\widetilde{N_{1}} \# N_{2} \# \widetilde{N_{1}}\right) \cong \mathbb{Z}_{2}$ but $H_{1}\left(g_{0}^{*}\right) \cong \mathbb{Z}$ and the number of $\widetilde{N_{1}}$ in $g_{0}^{*}$ exceeds the number of $\widetilde{N}_{1}$ in $N_{K}$.

Theorem 2.4. Let $N=N_{1} \# N_{2}$ be a closed s-Hopfian $n$-manifold $(n \geq 5)$ with nontrivial Hopfian $\pi_{1}\left(N_{i}\right)$ for $i=1,2$. Then $N$ is a codimension-2 fibrator if and only if $N$ is not the homotopy type of $\mathbb{R} P^{n} \# \mathbb{R} P^{n}$.

Proof. $(\Rightarrow)$ Any closed manifold that cyclically covers itself nontrivially fails to be a codimension-2 fibrator [6, Theorem 4.2].

$(\Leftarrow)$ We only need to consider the case that $\pi_{1}\left(N_{i}\right) \cong \mathbb{Z}_{2}(i=1,2)$, otherwise $\pi_{1}(N)$ is hyperhopfian. Suppose that $N$ is not the homotopy type of $\mathbb{R} P^{n} \# \mathbb{R} P^{n}$. Say $N_{1} \nsucceq \mathbb{R} P^{n}$.

Let $p: M \longrightarrow B$ be an $N$-like proper map defined on an $(n+2)$-manifold $M$. Put $G=\left\{p^{-1}(b): b \in B\right\}$. Once localizing the situation in light of Proposition 1.2, then applying to Lemma 2.2 twice, we see that $p$ is an approximate fibration over int $B$. 
Just mimicking the method of the proof in [16, Lemma 3.2], we can conclude that $\partial B=\emptyset$. For the sake of completeness, we give the proof $\partial B=\emptyset$ as follows:

Suppose $\partial B \neq \emptyset$. Then there exist $z_{0} \in \partial B$, a neighborhood $U$ of $z_{0}$ in $B$, $U \cap\left(\right.$ int $\left.B \backslash C^{\prime}\right)=\emptyset$, and a deformation retraction $R: p^{-1}(U) \rightarrow p^{-1}\left(z_{0}\right)$ satisfying

(1) $U$ is homeomorphic to the upper half plane $\mathbb{R}_{+}^{2}=\left\{(x, y) \in \mathbb{R}^{2} \mid y \geq 0\right\}$,

(2) $A=U \cap \partial B$ is an open arc,

(3) for all $z \in L, R \mid p^{-1}(z): p^{-1}(z) \rightarrow p^{-1}\left(z_{0}\right)$ is a homotopy equivalence, and

(4) $p$ is an approximate fibration over int $U$ (see [6]).

Take the covering map $q: M_{H} \longrightarrow p^{-1}(U)$ corresponding to $H=\bigcap_{i \in I}\left\{H_{i}\right.$ : $\left.\left[\pi_{1}(N): H_{i}\right]=2\right\}$. Then we have that for all $a \in A, q^{-1}\left(p^{-1}(a)\right)$ is connected and $q^{-1}\left(p^{-1}(a)\right) \simeq q^{-1}\left(p^{-1}\left(a_{0}\right)\right) \simeq N_{H}$. Also, since $p$ is an approximate fibration over $p^{-1}($ int $U)$, for all $b, b^{\prime} \in$ int $U, q^{-1}\left(p^{-1}(b)\right)_{C} \simeq q^{-1}\left(p^{-1}\left(b^{\prime}\right)\right)_{C} \simeq$ (say) $N_{K}$, where $q^{-1}\left(p^{-1}(b)\right)_{C}$ and $q^{-1}\left(p^{-1}\left(b^{\prime}\right)\right)_{C}$ are components of $q^{-1}\left(p^{-1}(b)\right)$ and $q^{-1}\left(p^{-1}\left(b^{\prime}\right)\right)$, respectively, and $N_{K}$ is the covering of $N$ determined by a subgroup $K$ of $\pi_{1}(N)$. Hence, by the fact that $M_{H}$ is orientable and [6. Proposition 2.9], we see that for all $b \in$ int $U$ and for all $a \in A$, the components $q^{-1}\left(p^{-1}(b)\right)_{C}$ of $q^{-1}\left(p^{-1}(b)\right)$ and $q^{-1}\left(p^{-1}(a)\right)$ are orientable. Therefore, if $G^{*}=\left\{q^{-1}\left(p^{-1}(b)\right)_{C}, q^{-1}\left(p^{-1}(a)\right) \mid b \in\right.$ int $U, a \in A\}$ is the usc decomposition of $M_{H}$, then $B^{*}=M_{H} / G^{*}$ is a 2-manifold without boundary. Let $p^{*}: M_{H} \longrightarrow B^{*}$ be the decomposition map and $C^{*}$ be its continuity set. Since $p^{*}\left(q^{-1}\left(p^{-1}(A)\right)\right)$ is homeomorphic to an open $\operatorname{arc}$ and $B^{*} \backslash C^{*}$ is locally finite in $B^{*}$, there is a point $a^{*} \in p^{*}\left(q^{-1}\left(p^{-1}(A)\right)\right) \cap C^{*}$. So we have a degree one map $N_{K} \simeq q^{-1}\left(p^{-1}(b)\right)_{C} \longrightarrow q^{-1}\left(p^{-1}(a)\right) \simeq N_{H}$ with $p^{*}\left(q^{-1}\left(p^{-1}(a)\right)\right)=a^{*}$ and for some $b \in$ int $U$. This is impossible, unless $K=H$.

Note. We do not exclude the case of $\pi_{1}\left(N_{1}\right)=1$ or $\pi_{1}\left(N_{2}\right)=1$ in Theorem 2.2 while we do exclude those cases in Theorem 2.4.

Remark. Theorem 2.4 can be restated as follows:

Let $N=N_{1} \# N_{2}$ be a closed s-Hopfian $n$-manifold $(n \geq 5)$ with nontrivial Hopfian $\pi_{1}\left(N_{i}\right)$ for $i=1,2$. Then $N$ is a codimension-2 fibrator if and only if $N$ does not regularly and cyclically cover itself, up to homotopy type.

The condition that $N$ does not regularly and cyclically cover itself, up to homotopy type, is also a necessary and sufficient condition for s-Hopfian manifolds to be codimension-2 fibrators in some non-connected sum case as follows:

Proposition 2.5. Suppose $N$ is a closed aspherical manifold and $\pi_{1}(N)$ is a residually finite group with finite abelianization. If $N$ fails to be a codimension-2 fibrator, then $N$ regularly and cyclically covers itself, up to homotopy type.

Proof. If $N$ fails to be a codimension-2 fibrator, we have a map $f: N \rightarrow N$ with $f_{\#}\left(\pi_{1}(N)\right) \neq \pi_{1}(N), f_{\#}\left(\pi_{1}(N)\right) \triangleleft \pi_{1}(N)$ and $\pi_{1}(N) / f_{\#}\left(\pi_{1}(N)\right)$ finite cyclic 6]. Then $f_{\#}$ is monomorphism by Hirshon's result [14, Corollary 3]. Take a covering $\theta: N_{f_{\#}\left(\pi_{1}(N)\right)} \rightarrow N$ determined by $f_{\#}\left(\pi_{1}(N)\right)$, and pass to the lifting $\bar{f}: N \rightarrow N_{f_{\#}\left(\pi_{1}(N)\right)}$ so that $\theta \circ \bar{f}=f$. Then $\bar{f}_{\#}$ is an isomorphism, for $f_{\#}$ is monomorphism. Since $N$ is aspherical, $f$ is a homotopy equivalence.

\section{REFERENCES}

[1] N. Chinen, Finite groups and approximate fibrations, Topology Appl. 102 (2000) 59-88 CMP 2000:08

[2] S.E. Cappell and J.L. Shaneson, Some new four-manifolds, Ann. of Math. (2) 104 (1976) 61-72 MR 54:6167 
[3] D.S. Coram and P.F. Duvall, Approximate fibration, Rocky Mountain J. Math. 7 (1977) 275-288 MR 56:1296

[4] D.S. Coram and P.F. Duvall, Approximate fibration and a movability condition for maps, Pacific J. Math. $\mathbf{7 2}$ (1977) 41-56 MR 57:7597

[5] D.S. Coram and P.F. Duvall, Mappings from $S^{3}$ to $S^{2}$ whose point inverses have the shape of a circle, General Topology Appl. 10 (1979) 239-246 MR 81b:57009

[6] R.J. Daverman, Submanifold decompositions that induce approximate fibrations, Topology Appl. 33 (1989) 173-184 MR 91d:57013

[7] R.J. Daverman, Hyper-Hopfian groups and approximate fibrations, Compositio Math. 86 (1993) 159-176 MR 94b:55022

[8] R.J. Daverman, Codimension-2 fibrators with finite fundamental groups, Proc. Amer. Math. Soc. 127 (1999) 881-888 MR 2000a:57051

[9] R.J. Daverman and Y. Kim, 2-groups and approximate fibrations, Topology Appl. To appear

[10] R.J. Daverman, Y.H. Im and Y. Kim, Products of Hopfian manifolds and codimension-2 fibrators, Topology Appl. 103 (2000) 323-338 CMP 2000:12

[11] R. Fintushel and R.J. Stern, Smooth free involutions on homotopy $4 k$-spheres, Michigan Math. J. 30 (1983) 37-51 MR 84f:57025

[12] J.A. Hillman, The algebraic characterization of geometric 4-manifolds, Cambridge Univ. Press, Cambridge, 1994 MR 95m:57032

[13] J.A. Hillman, 2-knots and their groups, Cambridge Univ. Press, Cambridge, 1989 MR 90d:57025

[14] R. Hirshon, Some properties of endomorphisms in residually finite groups, J. Austral. Math. Soc. Series A 34 (1977), 117-120. MR 57:9847

[15] Y.H. Im and Y. Kim, Hopfian and strongly hopfian manifolds, Fund. Math. 159 (1999) 127-134 MR 99j:57023

[16] Y. Kim, Strongly Hopfian manifolds as codimension-2 fibrators. Topology Appl. 92 (1999) 237-245 MR 2000g:57036

[17] Y. Kim, Connected sums of manifolds which induce approximate fibrations, Proc. Amer. Math. Soc. 128 (2000) 1497-1506 MR 2000j:57052

[18] Y. Kim, Manifolds with hyper-Hopfian fundamental group as codimension-2 fibrators, Topology Appl. 96 (1999) 241-248 CMP 2000:01

[19] S. Lopez de Medrano, Involutions on manifolds, Springer-Verlag, New York, (1971) MR 45:7747

Department of Mathematics, Pusan National University, Pusan, 609-735, Korea

E-mail address: yhim@hyowon.pusan.ac.kr

Department of Mathematics, Kyungpook National University, Taegu, 702-701, Korea

E-mail address: yongkuk@knu.ac.kr 